those individuals with 'between episode' behavioural abnormalities to avoid a legalistic haven for habitually violent individuals. It seems difficult to differentiate between episodes of dyscontrol and 'between episode' aggressiveness. The criteria are, however, consistent with the current view that individuals with antisocial personalities are in the main not treatable and, therefore, not part of the work of psychiatrists.

Disappointingly, despite a considerable number of studies showing a reduction of aggressive behaviour linked to anticonvulsant treatment, anticonvulsants have not been widely used in the absence of definite brain damage. I would suggest that some people do have episodes of violent behaviour mediated by limbic dysfunction of some kind, but that they rapidly develop a range of secondary difficulties in the areas of education, employment, relationships, etc. It then becomes impossible to define them as a group, as they appear identical to individuals with antisocial personality disorder.

MALETSKY, B. M. (1973) The episodic dyscontrol syndrome. Diseases of the Nervous System, 34, 178-185.

\section{Rampton Hospital}

Retford

Nottinghamshire DN22 OPD

Simon D. PAYNE

\section{SSRIs and tricyclic antidepressants}

SIR: The case studies of Seth et al (Journal, October 1992, 161, 562-565) suggesting combination studies with 5-HT reuptake inhibitor and noradrenergic tricyclic antidepressant (with or without concurrent lithium therapy) appear to offer new hope for the treatment of resistant depression, especially in elderly patients.

It must be remembered that 5-HT reuptake inhibitors are pharmacologically distinct and a heterogeneous group of drugs. The toxicity of tricyclic antidepressants is plasma-dependent (Preskorn, 1991). Certain 5-HT reuptake inhibitors are known to inhibit tricyclic antidepressant metabolism; paroxetine ( $30 \mathrm{mg}$ per day) causes a three-fold increase in half-life and a five-fold increase in the clearance serum desipramine (Brosen et al, 1992). There are a number of case reports testifying to the clinical relevance of interaction between fluoxetine and tricyclic antidepressant levels (e.g. Brosen \& Skelbo, 1990). Sertraline appears to have less effect in inhibiting the metabolism of tricyclic antidepressants. There is some indication that an antidepressant-free interval may be necessary when switching from fluoxetine to a tricyclic, if one is to be cautious to avoid potentially toxic tricyclic plasma levels (van Ameringen \& Mancini, 1992).

The Committee on Safety of Medicines (1989) warned that the combinations of selected 5-HT reuptake inhibitors with monoamine oxidase inhibitors, lithium, or tryptophan, while potentially beneficial in some selected cases, can increase the severity of 5-HT-related side-effects. In the most severe cases (life-threatening 5-HT syndrome of hypothermia), tremor convulsions may develop.

In the management of elderly depressed people who may often have concomitant physical illness, serious consideration must be given when choosing a selective 5-HT reuptake inhibitor for use in combination therapy, with a clear understanding of the potential risks associated with the various drugs.

Brosen, K., et al (1992) Pharmacogenetics of tricyclic antidepressants and novel antidepressants: recent developments. Clinical Neuropharmacology, 15, 80A-81A.

- \& SKelbo, E. (1990) Fluoxetine and norfluoxetine are potent inhibitors of P450-II-D6 - the source of the sparteine/ debrisoquine oxidation polymorphism. British Journal of Clinical Pharmacology, 32, 136-137.

Committee on SAfeTy of Medicines (1989) Current Problems (May).

Preskorn, S. H. \& FAST, G. A. (1991) Therapeutic drug monitoring for antidepressants: efficacy, safety and cost effectiveness. Journal of Clinical Psychiatry, 52, 23-33.

van Ameringen, M. \& Mancini, C. (1992) Adverse effects of switching from fluoxetine to desipramine. Canadian Journal of Psychiatry, 37, 278.

L. J. ASHWORTH

The University of Liverpool

K. C. M. WILSON

Department of Psychiatry

P.O. Box 147

Liverpool L69 3BX

\section{HTLV-1 Revisited}

SIR: In view of the continuing investigation into the aetiology of psychotic illness, we wish to report our findings on the seroprevalence of human $\mathrm{T}$-cell lymphotropic virus-1 (HTLV-1) in a group of acute psychiatric patients in Trinidad and Tobago.

HTLV-1 is endemic to Trinidad and Tobago with a reported seroprevalence of $1.6 \%$ among the healthy population (Daisley et al, 1991). Retroviral infection has been suggested as a possible aetiological mechanism in psychosis, especially since retroviruses can be incorporated into the human genome (Crow, 1984). Although previous studies have not found a demonstrable link between retroviral infection and schizophrenia (Feenstra et al, 1989), we felt that because HTLV-1 is endemic to our population, the 